\title{
Resonant tunnel magnetoresistance in double-barrier planar magnetic tunnel junctions
}

\author{
A. N. Useinov* and J. Kosel \\ Sensing, Magnetism, and Microsystems Group, Division of Physical Sciences and Engineering, King Abdullah University of Science and \\ Technology, Thuwal 23955-6900, Saudi Arabia \\ N. Kh. Useinov and L. R. Tagirov \\ Solid State Physics Department, Kazan Federal University, Kazan 420008, Russia \\ (Received 20 February 2011; revised manuscript received 30 May 2011; published 24 August 2011)
}

\begin{abstract}
We present a theoretical approach to calculate the spin-dependent current and tunnel magnetoresistance (TMR) in a double-barrier magnetic tunnel junction (DMTJ), in which the magnetization of the middle ferromagnetic metal layer can be aligned parallel or antiparallel in relation to the fixed magnetizations of the left and right ferromagnetic electrodes. The electron transport through the DMTJ is considered as a three-dimensional problem, taking into account all transmitting electron trajectories as well as the spin-dependent momentum conservation law. The dependence of the transmission coefficient and spin-polarized currents on the applied voltage is derived as an exact solution to the quantum-mechanical problem for the spin-polarized transport. In the range of the developed physical model, the resonant tunneling, nonresonant tunneling, and enhanced spin filtering can be explained; the simulation results are in good agreement with experimental data.
\end{abstract}

DOI: 10.1103/PhysRevB.84.085424

PACS number(s): 75.45.+j, 72.10.Fk, 75.47.De, 73.40.Gk

\section{INTRODUCTION}

Spintronics is a rapidly developing branch of nanoscience and nanotechnology, with many applications in electronics. Ferromagnetic alloys are the natural media for electrons with both kinds of spins and nonequal transport properties. The resonant transparency of a double-barrier magnetic tunnel junction (DMTJ) may occur at very different applied voltages for the spin-up and spin-down electrons, due to high exchange energy splitting of the electron conduction bands (in $\mathrm{Fe}$, $\mathrm{FeCoB}$, and $\mathrm{FeNi}$ alloys). As a result, high spin filtering or spin selection effects are expected in DMTJ. Assuming that during the resonant tunneling process the electron spins are conserved, the tunneling of spin-up and spin-down electrons can be analyzed within the model of two conduction channels, ${ }^{1-3}$ where electrons originating from a particular spin state of the left spin-polarized electrode tunnel into the empty states of the right electrode (Fig. 1 and Fig. 2). It was shown ${ }^{4-7}$ that DMTJ can provide the function of a memory cell (MRAM) or spin-current diode, with possible applications in electronic and spintronic devices.

DMTJs were studied experimentally with possible applications $^{4-12}$ and simulated theoretically in several cases. ${ }^{13-20}$ Among the theoretical studies, combinations of different materials for single- and double-barrier structures can be found. For example, heterostructures with semiconductor electrodes on both sides and a central semiconductor quantum well $(\mathrm{QW})^{13}$ or ferromagnetic and hybrid semiconductor/ferromagnetic DMTJ systems ${ }^{14,19}$ have been studied. Most of these works are based on the Landauer-Büttiker formalism, the tunnel Hamiltonian treatment, or the well-known freeelectron tunneling model of Tsu and Esaki, ${ }^{21}$ which for the first time generalized a theory for multibarrier tunnel structures. Based on Esaki's model, Zhang et al. ${ }^{14}$ developed a transfer-matrix treatment and a two-band model with dc bias. However, the calculation of tunnel currents was limited to low temperatures and a small thicknesses of insulating barriers (around $5 \AA$ ).
Zhang ${ }^{14}$ and Kishi et al.${ }^{15}$ were among the first who theoretically investigated conductance and tunnel magnetoresistance (TMR) properties for the DMTJ while taking into account the electric field inside the insulators and with the ultrathin ferromagnetic middle layer acting as a quantum well. They pointed out that the tunneling conductance oscillates, showing peaks at resonant voltages, which lead to an enhanced TMR effect.

Important theoretical studies were made by Wilczynski et al. in Ref. 16, where DMTJ was built with two ferromagnetic electrodes sandwiching the middle nonmagnetic layer and studied within the free-electron approximation. It was assumed that the sequential tunneling regime in such structures could be realized; however, transmission coefficients (TC) through the left and right barriers were calculated separately.

Previously, we developed a theory of electronic transport through a nanosized domain wall between ferromagnetic homo- and hetero-electrodes ${ }^{22}$ in a pointlike geometry. This theory utilizes quasiclassical equations in order to describe the transport phenomena in the ferromagnetic metals, and quantum-mechanical equations for modeling the electron domain wall, which is approximated by a slope potential. In this work, we apply the previously derived equation for the current $^{22}$ to the case of electronic transport through the DMTJ in a ballistic mode replacing the transmission coefficient of the domain wall potential by the tunnel transmission coefficient (which is calculated analytically for the DMTJ). The inhomogeneous terms (responsible for the pointlike geometry) were neglected due to their small contribution in the planar ballistic case. Thus, the DMTJ theory is extended toward high Fermi energies $(\sim 5 \mathrm{eV})$, accounting for the dependence of the electron transmission on the trajectory angle (Fig. 1) and the conduction band spin splitting (Fig. 2), while assuming that the electron spin is conserved during the tunneling process and maintaining the traditional free-electron parabolic dispersion relation. This provides an alternative view on the fundamental 


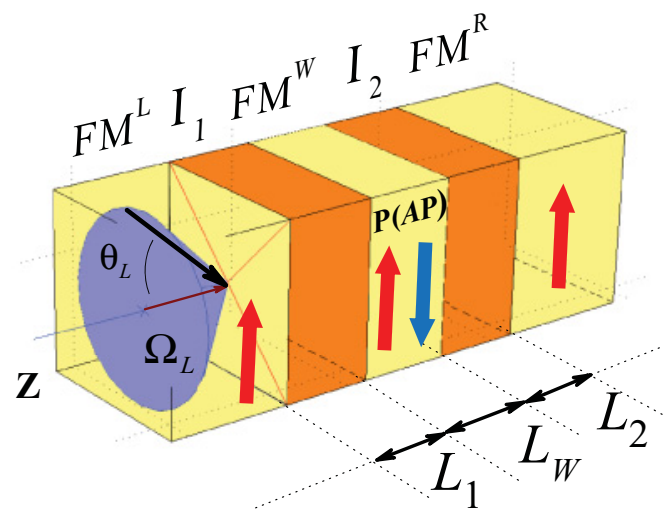

FIG. 1. (Color online) Schematic view of the planar DMTJ. Thick red and blue arrows show possible magnetization directions of the ferromagnetic layers. $L_{1(2)}$ are the insulator thicknesses, and $L_{W}$ is the width of the middle ferromagnetic layer $\mathrm{FM}^{\mathrm{W}}$. The black arrow inside the cone within the solid angle $\Omega_{L}$ indicates the direction of the electron trajectory, which has the trajectory angle $\theta_{L}$ with the $z$ axis.

aspects of contemporary attempts to construct spin-valve magnetic tunnel junction devices ${ }^{6,7}$ as well as a self-consistent method for image detection in scanning tunnel microscopy with ferromagnetic cantilevers. ${ }^{23,24}$

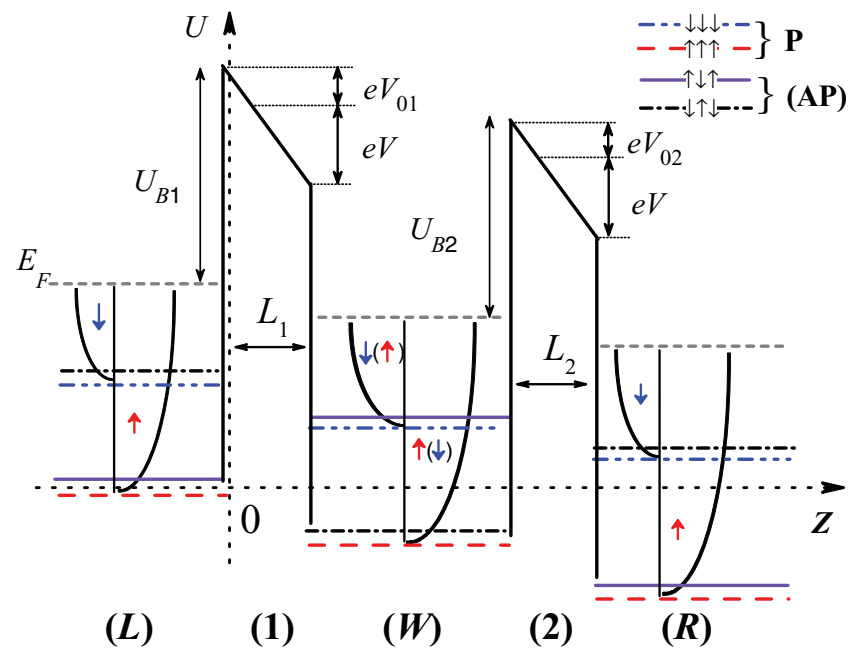

FIG. 2. (Color online) Schematic energy diagram for the potential profile of the $\mathrm{FM}^{\mathrm{L}} / \mathrm{I}_{1} / \mathrm{FM}^{\mathrm{W}} / \mathrm{I}_{2} / \mathrm{FM}^{\mathrm{R}}$ DMTJ with applied bias $V_{a}$, where $V$ are voltage drops on the first and second barriers $\left(V_{a}=2 V\right)$. The $U_{B 1(2)}$ are the heights of the barriers above $E_{\mathrm{F}}$. The enhanced parabolic curves present dispersion relations for spin-up electrons $(\uparrow$ arrows) and correspond to the spin-up majority conductance subbands for the parallel $(\mathrm{P})$ case. The small parabolic curves belong to the spin-down minority subbands with spin-down electrons ( $\downarrow$ arrows). The arrows inside the brackets of the middle layer show the electron spin direction for the antiparallel (AP) case. The electron spinconduction channels passing through minority or majority subbands are determined by the electron-tunneling trajectory with conserved spin direction. They are shown at the bottoms of the subbands as blue dash-dot-dotted lines and red dashed lines for the P case and as dash-dotted and solid lines for the AP case.

\section{TUNNELING CURRENT AND TRANSMISSION COEFFICIENT FOR DMTJ}

We consider a spin-dependent resonant and nonresonant tunneling effect in a DMTJ consisting of $\mathrm{FM}^{\mathrm{L}} / \mathrm{I}_{1} / \mathrm{FM}^{\mathrm{W}} / \mathrm{I}_{2} / \mathrm{FM}^{\mathrm{R}}$, where spin-dependent $\mathrm{QW}$ states created in the ultrathin $\mathrm{FM}^{\mathrm{W}}$ layer provide different tunneling conditions for $\mathrm{P}$ and AP cases. Upon derivations, it is assumed that the Fermi energy of the conduction electrons in metals $E_{\mathrm{F}}$, exchange energy $E_{\mathrm{ex}}$, barrier heights $U_{B 1(2)}$, and applied voltage $e V_{a}$ (see Fig. 2) are much larger than the thermal energy $k_{\mathrm{B}} T$. The ferromagnetic layers $\mathrm{FM}^{\mathrm{L}}, \mathrm{FM}^{\mathrm{W}}$, and $\mathrm{FM}^{\mathrm{R}}$ can be made of different ferromagnetic metals characterized by effective masses of conduction electrons, Fermi energies, and spin polarizations of the conduction band.

The schematic potential diagrams with conduction band locations for spin-up and spin-down electrons are shown in Fig. 2. The $z$ axis is perpendicular to the junction, and the interface between the left electrode and the first barrier is located at $z=0$. Both barriers are supposed to be identical so that the applied positive voltage $2 \mathrm{~V}$ is divided equally between barriers (see Fig. 2).

In the free-electron model of the spin-polarized conduction electrons the tunnel current per unit area of a planar junction for the conduction channel is proportional to the product of the transmission coefficient and cosine of the incidence angle $\theta_{L, s}$ of the electron trajectory, averaged over the solid angle $\Omega_{L}$ (Fig. 1) of the left electrode (the electron spin is assumed conserved during the transmission through the DMTJ): ${ }^{25}$

$$
J_{s}^{\mathrm{P}(\mathrm{AP})}=\frac{e^{2}\left(k_{F, s}^{L}\right)^{2} V_{a}}{4 \pi^{2} \hbar}\left\langle\cos \left(\theta_{L, s}\right) D_{s}^{\mathrm{P}(\mathrm{AP})}\right\rangle_{\Omega_{L}},
$$

where $k_{F, S}^{L}$ is the absolute value of the Fermi wave vector of the electron in the left electrode for the spin channels $s=\uparrow, \downarrow$ for the $\mathrm{P}$ and $\mathrm{AP}$ cases. $D_{s}^{\mathrm{P}(\mathrm{AP})}$ is the transmission coefficient through the double barrier, which is a function of the applied voltage $V_{a}=2 \mathrm{~V}$ and $\cos \left(\theta_{L, s}\right)$. The angle $\theta_{L, s}$ is measured from the normal to the contact plane, and the angle $\phi$ is in the contact plane. The integration by solid angle in spherical coordinates is

$$
\begin{aligned}
\langle(\ldots)\rangle_{\Omega_{L}} & \equiv \frac{1}{2 \pi} \int d \Omega_{L}(\ldots) \\
& =\frac{1}{2 \pi} \int_{0}^{\theta_{C R}} \sin \left(\theta_{L, s}\right) d \theta_{L, s} \int_{0}^{2 \pi} d \phi(\ldots),
\end{aligned}
$$

where $\theta_{C R}$ is the critical angle. It is defined by the conservation law of the Fermi wave vector projection on the junction plane (longitudinal component):

$$
\begin{aligned}
k_{L, s}^{\|} & =k_{F, s}^{L} \sin \left(\theta_{L, s}\right) \\
& =k_{F, s}^{W}(V) \sin \left(\theta_{W, s}\right)=k_{F, s}^{R}(V) \sin \left(\theta_{R, s}\right) .
\end{aligned}
$$

Here $k_{F, s}^{W}(V)=\sqrt{\left(k_{F, S}^{W}\right)^{2}+c_{W} V}$ and $k_{F, s}^{R}(V)=\sqrt{\left(k_{F, S}^{R}\right)^{2}+c_{R} V}$ are the bias-dependent absolute values of the Fermi wave vectors of the middle $\mathrm{FM}^{\mathrm{W}}$ and right $\mathrm{FM}^{\mathrm{R}}$ layers, where $c_{W(R)}=$ $2 m_{W(R)} e / \hbar^{2}$ are dimensional factors. For the sake of simplicity, we denote this factor as $c_{l}=2 m_{l} e / \hbar^{2}$, where $m_{l}$ stands for the effective masses of the electron in the five regions (Fig. 2) labeled as $\mathrm{FM}^{\mathrm{L}}, \mathrm{I}_{1}, \mathrm{FM}^{\mathrm{W}}, \mathrm{I}_{2}$, and $\mathrm{FM}^{\mathrm{R}}$ in Fig. 1. The angles $\theta_{l, s}(l=L, W, R)$ in Eq. (3) are measured from the normal to 
the contact plane. The transverse components (parallel to the $z$ axis) of the wave vectors for each ferromagnetic layer are as follows:

$$
\begin{gathered}
k_{L, s}^{\perp}=k_{F, s}^{L} \cos \left(\theta_{L, s}\right), \\
k_{W, s^{\prime}}^{\perp}=k_{F, s^{\prime}}^{W}(V) \cos \left(\theta_{W, s^{\prime}}\right), \\
k_{R, s}^{\perp}=k_{F, s}^{R}(V) \cos \left(\theta_{R, s}\right),
\end{gathered}
$$

where $s$ and $s^{\prime}$ are the indices showing the type of the spin subband (minority $s=\downarrow, s^{\prime}=\downarrow, s=\downarrow$, or majority $s=\uparrow, s^{\prime}=\uparrow, s=\uparrow$ ), which contributes to the spin-current channels; specifically, in the case of a $\mathrm{P}$ alignment of the magnetization of the electrodes $s=\uparrow(\downarrow), s^{\prime}=\uparrow(\downarrow), s=\uparrow$ $(\downarrow)$ and for the AP alignment $s=\uparrow(\downarrow), s^{\prime}=\downarrow(\uparrow), s=\uparrow(\downarrow)$.

In order to execute averaging over the solid angle $\Omega_{L}$ in Eq. (1), it is necessary to express the $\cos \left(\theta_{W, s}\right)$ and $\cos \left(\theta_{R, s}\right)$ in Eq. (4) in terms of the cosine of the left angle $\theta_{L, s}$ (see Fig. 1) using the conservation law for the longitudinal projection of the momentum (3):

$$
\begin{aligned}
& \cos \left(\theta_{W, s}\right)=\sqrt{1-\delta_{L W}^{2}\left[1-\cos ^{2}\left(\theta_{L, s}\right)\right]}, \\
& \cos \left(\theta_{R, s}\right)=\sqrt{1-\delta_{L R}^{2}\left[1-\cos ^{2}\left(\theta_{L, s}\right)\right]}
\end{aligned}
$$

where $\delta_{L W}=k_{F, S}^{L} / k_{F, S}^{W}(V), \delta_{L R}=k_{F, s}^{L} / k_{F, S}^{R}(V)$ are the spinasymmetry parameters of the conductance channels for the first and second barriers, respectively. Equations (5) determines the value of the critical angle for $\theta_{L, s}$. Thus, the value $\theta_{C R}$ in Eq. (2) is taken as $\min \left\{\theta_{1}, \theta_{2}\right\}$ with $\theta_{1}=\arccos \left(\sqrt{\left|1-1 / \delta_{L W}^{2}\right|}\right)$ and $\theta_{2}=\arccos \left(\sqrt{\left|1-1 / \delta_{L R}^{2}\right|}\right)$.

The transmission coefficient is defined in a standard way as a ratio of the transmitted flux of probability density $j_{R, s}$, in $\mathrm{FM}^{\mathrm{R}}$ to the incident one in the $\mathrm{FM}^{\mathrm{L}}, j_{L, s}$. Assuming that the wave function amplitude in the $\mathrm{FM}^{\mathrm{L}}$ layer equals unity, and there is only the transmitted component of $j_{R, s}$ in $\mathrm{FM}^{\mathrm{R}}$, the transmission coefficient $D_{s}^{\mathrm{P}(\mathrm{AP})}$ can be written as follows:

$$
D_{s}^{\mathrm{P}(\mathrm{AP})}=\frac{m_{L} k_{R, s}^{\perp}}{m_{R} k_{L, s}^{\perp}}\left(\Psi_{s}^{\mathrm{P}(\mathrm{AP})} \Psi_{s}^{\mathrm{P}(\mathrm{AP})^{*}}\right),
$$

where $\Psi_{s}^{\mathrm{P}(\mathrm{AP})}$ is the complex-valued amplitude of the wave function of transmitted electron with spin $s$.

In this paper, we deduce an expression for $\Psi_{s}^{\mathrm{P}(\mathrm{AP})}$ by solving the Schrödinger equations in the $\mathrm{FM}^{\mathrm{L}} / \mathrm{I}_{1} / \mathrm{FM}^{\mathrm{W}} / \mathrm{I}_{2} / \mathrm{FM}^{\mathrm{R}}$ sandwich using a convenient matrix form proposed in Ref. 26. The dependence of the transverse components of the wave vectors on the angle $\theta_{L, s}$ is given by Eq. (4). The resulting expression for $\Psi_{s}^{\mathrm{P}(\mathrm{AP})}$ is as follows:

$$
\Psi_{s}^{\mathrm{P}(\mathrm{AP})}=\frac{4 m_{1} m_{W} m_{2} m_{R} e^{-i\left[\left(L_{1}+L_{2}+L_{W}\right) k_{R, s}^{\perp}-L_{W} k_{W, s}^{\perp}\right]} \rho_{1, s}^{\mathrm{P}(\mathrm{AP})} \rho_{2, s}^{\mathrm{P}(\mathrm{AP})} k_{L, s}^{\perp} k_{W, s}^{\perp}}{Z_{1, s}^{\mathrm{P}(\mathrm{AP})}+Z_{2, s}^{\mathrm{P}(\mathrm{AP})}},
$$

where

$$
\begin{aligned}
& Z_{1}=\left[m_{1} k_{L}\left(i m_{1} \alpha_{1} k_{W}+m_{W} \gamma_{1}\right)+m_{L}\left(m_{1} \beta_{1} k_{W}+i m_{W} \chi_{1}\right)\right]\left[m_{2} k_{R}\left(i m_{2} \alpha_{2} k_{W}+m_{W} \gamma_{2}\right)+m_{R}\left(m_{2} \beta_{2} k_{W}+i m_{W} \chi_{2}\right)\right] \\
& Z_{2}=\left[m_{1} k_{L}\left(m_{1} \alpha_{1} k_{W}+i m_{W} \gamma_{1}\right)-m_{L}\left(m_{W} \chi_{1}+i m_{1} \beta_{1} k_{W}\right)\right]\left[m_{2} k_{R}\left(m_{2} \alpha_{2} k_{W}+i m_{W} \gamma_{2}\right)-m_{R}\left(m_{W} \chi_{2}+i m_{2} \beta_{2} k_{W}\right)\right] e^{2 i L} k_{W}
\end{aligned}
$$

In Eq. (8), the indices P, AP, $s$, and $\perp$ are suppressed for simplicity. In addition to this, the following abbreviations are used:

$$
\begin{gathered}
\alpha_{1(2)}=\mathrm{Ai}\left(-q_{2(4)}\right) \mathrm{Bi}\left(-q_{1(3)}\right)-\mathrm{Ai}\left(-q_{1(3)}\right) \mathrm{Bi}\left(-q_{2(4)}\right), \\
\beta_{1(2)}=T_{1(2)}\left[\mathrm{Ai}^{\prime}\left(-q_{1(4)}\right) \mathrm{Bi}\left(-q_{2(3)}\right)-\operatorname{Ai}\left(-q_{2(3)}\right) \mathrm{Bi}^{\prime}\left(-q_{1(4)}\right)\right], \\
\gamma_{1(2)}=T_{1(2)}\left[\mathrm{Ai}^{\prime}\left(-q_{2(3)}\right) \mathrm{Bi}\left(-q_{1(4)}\right)-\mathrm{Ai}\left(-q_{1(4)}\right) \mathrm{Bi}^{\prime}\left(-q_{2(3)}\right)\right], \\
\rho_{1(2)}=T_{1(2)}\left[\mathrm{Ai}^{\prime}\left(-q_{2(4)}\right) \mathrm{Bi}\left(-q_{2(4)}\right)-\mathrm{Ai}\left(-q_{2(4)}\right) \mathrm{Bi}^{\prime}\left(-q_{2(4)}\right)\right], \\
\chi_{1(2)}=T_{1} T_{2}\left[\mathrm{Ai}^{\prime}\left(-q_{2(4)}\right) \mathrm{Bi}^{\prime}\left(-q_{1(3)}\right)-\mathrm{Ai}^{\prime}\left(-q_{1(3)}\right) \mathrm{Bi}^{\prime}\left(-q_{2(4)}\right)\right],
\end{gathered}
$$

where $\operatorname{Ai}\left(-q_{b}\right)$ and $\operatorname{Bi}\left(-q_{b}\right)$ are the Airy functions, while $\mathrm{Ai}^{\prime}\left(-q_{b}\right)$ and $\mathrm{Bi}^{\prime}\left(-q_{b}\right)$ are their first derivatives. The index $b$ runs from $b=1$ for the $\mathrm{FM}^{\mathrm{L}} / \mathrm{I}_{1}$ and $b=4$ for the $\mathrm{I}_{2} / \mathrm{FM}^{\mathrm{R}}$ interface. The arguments $q_{b}$ of the Airy functions are defined as follows:

$$
\begin{gathered}
q_{1}=T_{1} z_{L 1}, \\
q_{2}=T_{1}\left(L_{1}+z_{L 1}\right), \\
q_{3}=T_{2}\left(L_{1}+L_{W}+z_{L 2}\right), \\
q_{4}=T_{2}\left(L_{1}+L_{W}+L_{2}+z_{L 2}\right),
\end{gathered}
$$

where $L_{1(2)}$ are the barriers' thicknesses, and $L_{W}$ is the width of the middle ferromagnetic layer. The other quantities $T_{1(2)}$ and $z_{L 1(2)}$ in Eq. (10) are given by

$$
\begin{gathered}
T_{1(2)}=\left(\frac{c_{1(2)} V_{1(2)}}{L_{1(2)}}\right)^{1 / 3}, \\
z_{L 1}=\frac{\left(k_{F, V_{a}=0}^{L}\right)^{2}-c_{L} u_{1}}{c_{L} V_{1}} L_{1}, \\
z_{L 2}=\frac{\left(k_{F, V_{a}=0}^{W}\right)^{2}-c_{W} u_{2}}{c_{W} V_{2}} L_{2}+L_{2}-L_{1}-L_{W},
\end{gathered}
$$

where $V_{1(2)}=V_{01(2)}+V$ is the sum of the intrinsic voltage $V_{0}$ at zero bias and the voltage drop $V$, and $u_{1(2)}=$ $\left(E_{\mathrm{F}}+U_{B 1(2)}\right) / e$ is the potential barrier height. The voltage $V_{0}$ is rather small $(0.01-0.1 \mathrm{~V})$ and arises from the chemical potential difference of the contacting electrodes. Now, with Eqs. (6)-(13), we are ready to analyze the spin-dependent current (1) as a function of the applied voltage, effective masses, heights of the barriers, and exchange splitting of the conduction band in the ferromagnetic electrodes. Compared to 
similar studies in Refs. 14,16 we also included explicitly the dependence on the electron trajectory angle $\theta_{L}$.

\section{RESONANT TUNNEL MAGNETORESISTANCE IN DMTJ}

The TMR of DMTJ is defined as the change of the current upon transition from $\mathrm{P}$ to AP alignment of the magnetization of the middle ferromagnetic metal layer $\mathrm{FM}^{\mathrm{W}}$ in relation to the fixed magnetizations of the left $\mathrm{FM}^{\mathrm{L}}$ and right $\mathrm{FM}^{\mathrm{R}}$ ferromagnetic electrodes,

$$
\mathrm{TMR}=\frac{J^{\mathrm{P}}-J^{\mathrm{AP}}}{J^{\mathrm{AP}}},
$$

where $J^{\mathrm{P}(\mathrm{AP})}$ are the net current densities $\left(J_{\uparrow}^{\mathrm{P}(\mathrm{AP})}\right.$ plus $\left.J_{\downarrow}^{\mathrm{P}(\mathrm{AP})}\right)$ for the $\mathrm{P}(\mathrm{AP})$ cases.

For the numerical calculation, $\mathrm{FM}^{\mathrm{L}}$ and $\mathrm{FM}^{\mathrm{R}}$ were assumed to consist of the same ferromagnetic metals with identical physical parameters. The conduction band spin asymmetries in the left, right, and middle ferromagnetic electrodes are determined as $\delta_{L}=k_{F, \downarrow}^{L} / k_{F, \uparrow}^{L}, \delta_{R}=k_{F, \downarrow}^{R} / k_{F, \uparrow}^{R}$, and $\delta_{W}=$ $k_{F, \downarrow}^{W} / k_{F, \uparrow}^{W}$, respectively. For the middle $\mathrm{FM}^{\mathrm{W}}$ layer $\delta_{W}$ was assumed to be different from $\delta_{L}$ and $\delta_{R}$. Additionally, we assumed that for the paramagnetic limit (when the conduction band spin splittings vanish) the conduction subband bottoms in all FM layers would have the same positions at $V_{a}=0$. Two dielectric oxide layers $\left(\mathrm{Al}_{2} \mathrm{O}_{3}\right.$ or $\left.\mathrm{MgO}\right)$ with a lateral size comparable with the mean-free paths of the conduction electrons were considered as tunneling barriers. The potential profile of the system under the applied voltage $V_{a}$ is shown in Fig. 2, where $U_{B l}(l=1,2)$ are the barriers' heights above the Fermi energy. Symmetrical geometric structures with $L_{1}=L_{2}$ and $U_{B 1}=U_{B 2}$ were considered in all our calculations. However, due to a nonequal (compared with $\mathrm{FM}^{\mathrm{L}}$ and $\mathrm{FM}^{\mathrm{R}}$ ) conduction band spin asymmetry for the $\mathrm{FM}^{\mathrm{W}}$ layer the system is not symmetric in general.

First, the dependence of the transmission coefficient on the angle $\theta_{L, s}$ of the electron trajectory was considered (see Fig. 3) using the results of the previous section. The calculations have been carried out with the Fermi energy $E_{\mathrm{F}}=3.75 \mathrm{eV}$ for all ferromagnetic metal layers. The values of the Fermi wave vectors $k_{F, s}^{l}(l=L, R, W)$ for the FM layers were set to $k_{F, \uparrow}^{L(R)}=1.09 \AA^{-1}, k_{F, \uparrow}^{L(R)}=0.42 \AA^{-1}$ and $k_{F, \downarrow}^{W}=0.96 \AA^{-1}$, $k_{F, \uparrow}^{W}=1.0 \AA^{-1}$, respectively. The electron effective masses of the FM layers were taken equal to the free-electron mass $m_{e}$, while the electron effective masses in the insulating barriers $\mathrm{I}_{l}$ $(l=1,2)$ were taken as $m_{1(2)}=0.4 m_{e} \cdot{ }^{27}$ Further parameters were the middle layer thickness $L_{W}=13.6 \AA$, the thickness of the barriers $L_{1}=L_{2}=12 \AA$, and the barriers' heights $U_{B 1}=U_{B 2}=1.8 \mathrm{eV}$.

Figure 3 shows the plot of the transmission coefficient as a function of the voltage for two fixed trajectory angles: $\theta_{L, s}=$ $0.0^{\circ}$ (solid lines) and $\theta_{L, s}=10^{\circ}$ (dashed lines). The results show that inclination of the electron trajectory considerably influences the transmission coefficient.

At resonance, the values can increase significantly by up to 7 orders in relation to the nonresonant states for the whole range of the applied voltages. The resonant peaks, which correspond to the first and third spin channels, are

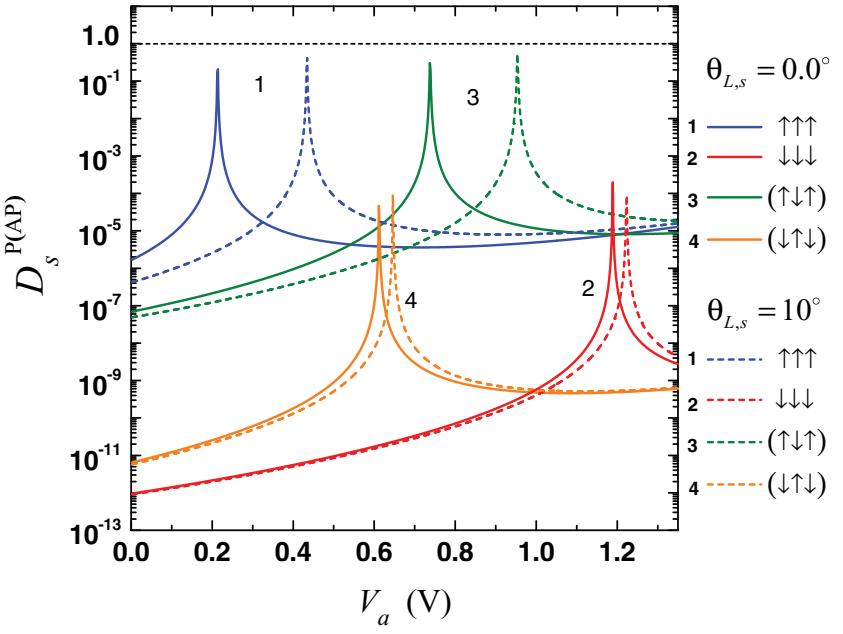

FIG. 3. (Color online) Transmission coefficients versus $V_{a}$ for four configurations of the conduction channels at each of the $\mathrm{P}$ and AP magnetic moment alignments and with two fixed trajectory angles: $\theta_{L, s}=0.0^{\circ}$ (solid lines) and $\theta_{L, s}=10^{\circ}$ (dashed lines). The parameters of the conduction bands' spin polarizations are $\delta_{L(R)}=$ $0.38, \delta_{W}=0.96$. Other parameters are as follows: $L_{1(2)}=12 \AA$, $L_{W}=13.6 \AA, U_{B 1(2)}=1.8 \mathrm{eV}, V_{01(2)}=0.1 \mathrm{eV}$, and $m_{1(2)}=0.4 m_{e}$.

by four orders larger than the ones of the second and fourth channels. Moreover, as $\theta_{L, s}$ increases to $10^{\circ}$ the positions of the peaks shift toward higher voltages. According to our results, for the most inclined electron trajectories with large $\theta_{L, s}$ [where tunneling conditions still apply, under conditions (5)] there is a nonresonant voltage interval (NRVI) $0.0-0.12 \mathrm{~V}$, where the transmission coefficients are small in relation to their peak values for all channels having values of $10^{-5}$ to $10^{-12}$ or smaller. As the voltage increases, NRVI increases too.

The restrictions by the angle, which stem from the momentum conservation law [see Eq. (3)], apply stringent conditions for the transmission and determine the values of the tunnel spin current.

It should be noted that TC depicted in Fig. 3 are particular cases for corresponding $L_{W}$, where there is at least one resonant peak for all spin channels in the voltage range $0.0<V_{a}<1.25 \mathrm{~V}$. The resonant peaks will not arise in this range of voltage if the DMTJ is simulated with another value of $L_{W}$. For example, for $L_{W}=12.5 \AA$ the NRVI increases to $0.0-1.22 \mathrm{~V}$, which is much larger than for $L_{W}=13.6 \AA$.

Thus, each spin-dependent conduction channel has its own constriction and resonance conditions. These conditions are most important for first and third channels, which provide the significant part in the tunnel current. This is the main reason for a giant TMR effect. Such tunneling is called spin-polarized resonant tunneling. ${ }^{14}$

In Fig. 4, the TMR is shown as a function of the thickness $L_{W}$ of the middle ferromagnetic layer in the case of $V_{a}=0.5 \mathrm{~V}$. The other parameters are the same as in Fig. 3. Some resonant features of the TMR curves are of particular interest: First, the TMR as a function of $L_{W}$ shows well-defined peaks, where the height of the peaks decreases monotonously with increasing $L_{W}$; and second, the TMR between peaks increases with increasing the electron effective masses $m_{1(2)}$ of the insulating barriers. TMR peaks periodically repeat every $3.1 \AA$ 


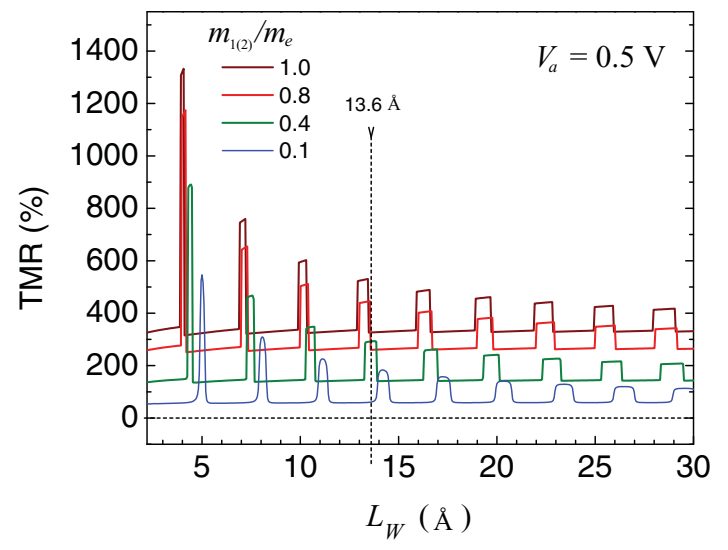

FIG. 4. (Color online) TMR as a function of the middle ferromagnetic layer $\mathrm{FM}^{\mathrm{W}}$ thickness $L_{W}$, calculated with the same parameters as in Fig. 3. The four curves correspond to different electron effective masses $m_{1(2)}$ of the insulating barriers.

corresponding to $k_{F, \uparrow}^{W}=1.0 \AA^{-1}$. The period of the peaks mainly depends on the values of $k_{F, \uparrow}^{W}$ : As $k_{F, \uparrow}^{W}$ decreases, the period increases; for $k_{F, \uparrow}^{W}=0.72 \AA^{-1}$ and $k_{F, \downarrow}^{W}=0.69 \AA^{-1}$ (keeping the same peak width) the peak period increases from $3.1 \AA$ to $4.0 \AA$. The width of the peaks mainly depends on $\delta_{W}$ (for $\delta_{W} \rightarrow 1$ the peak width vanishes). Moreover, it should be noted that the curve with $m_{1(2)}=m_{e}$ starting with the first peak of the TMR ratio at $1300 \%$ has peaks with sharper edges compared to the case when $m_{1(2)}=0.1 m_{e}$. The sharpness of the peak profile depends on $m_{1(2)}, E_{\mathrm{F}}$, and $U_{B 1(2)}$.

Thus, abrupt periodic variation of the TMR with increasing thickness $L_{W}$ is related both to the QW states formed in the middle ferromagnetic layer and to resonant tunneling through the whole structure. Similar results for a DMTJ structure with left/right ferromagnetic electrodes but a nonmagnetic middle layer have been shown previously. ${ }^{16}$ In this work the periodicity of the TMR magnitude was investigated for the first time, and it was found that TMR strongly correlates to QW states in the middle layer. The same finding is reproduced by our calculations.

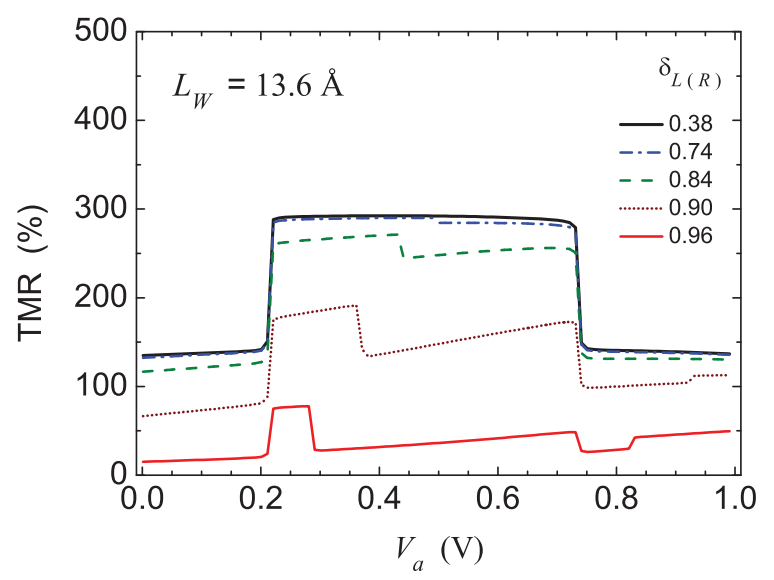

FIG. 5. (Color online) Resonant, flat-dome TMR behavior for different $\delta_{L(R)}\left(\delta_{R}=\delta_{L}\right)$ and fixed $\delta_{W}=0.96, m_{1(2)} / m_{e}=0.4$. The other parameters are the same as in Fig. 3.
The flat-dome TMR behavior, originating from the resonance tunneling, is shown in Fig. 5. Here, we investigate the dependence of TMR on spin asymmetry of the conduction band of the ferromagnetic layers when the thickness of the middle layer corresponds to the center of the flat bump in the TMR curve for $m_{1(2)}=0.4 m_{e}$ (see green line in the Fig. 4 , and vertical dashed line at $L_{W}=13.6 \AA$ ).

Figure 5 shows the dependence of the resonant behavior of the TMR on $V_{a}$ and $\delta_{L(R)}$. In the case of $\delta_{L(R)}=0.38$, the resonance arises as a flat-dome peak between $0.22<$ $V_{a}<0.72 \mathrm{~V}$ with a TMR value of $290 \%$ as compared to the nonresonant value of $135 \%$.

The beginning of the flat-dome TMR curve $\left(\delta_{L(R)}=0.38\right)$ depicted in Fig. 5 coincides with the location of the first resonant peak of the TC for the first channel in the $\mathrm{P}$ case for $V_{a} \approx 0.22 \mathrm{~V}$ (Fig. 3). The back edge of this flat-dome step localized at $V_{a} \approx 0.72$ corresponds to the AP resonant peak location for the third channel (see Fig. 3). This proves that the cause of the flat-dome shape is a result of resonant behavior of the electron tunneling for the particular data of the DMTJ with resonant width (which is one from the resonant width set; see Fig. 4). Moreover, it should be noted that with increasing $L_{W}$ the resonant TMR peaks may have a more complicated shape showing several flat-dome loops, which build on each other.

The resonance conditions arise in the cases when $U_{B 1(2)}>$ $1.35 \mathrm{eV}$ for $m_{L, W, R}=m_{e}$ and $U_{B 1(2)}>2.8 \mathrm{eV}$ for $m_{L, W, R}=$ $0.8 m_{e}$, while the TMR in NRVI is a result of coherent tunneling of the electrons through the barriers. As shown in Fig. 3 the TC dependencies in some range of the applied voltages are very small having values of $10^{-5}$ to $10^{-12}$ or smaller that are varying for different spin channels by several orders. This difference between spin channels provides the significant difference in the $I-V$ characteristics between the $\mathrm{P}$ and $\mathrm{AP}$ cases.

The same behavior of the TMR flat-dome peak (shown in Fig. 5) was predicted previously, ${ }^{17}$ where it was shown that a reduction of the dome width may occur with decreasing the parameter of the electronic correlation. In our case, it can be correlated with the barrier heights $U_{B 1(2)}$, but approximately the same effect can be achieved by a decrease of the spin polarization of the left and right electrodes. It should be noted that with decreasing the spin asymmetry of the conduction bands $\left(\delta_{L(R)}>0.74\right.$; see Fig. 5), the TMR flat dome becomes split in two steps with reduced amplitudes due to an increase of the peak values of the second and fourth channels. For $\delta_{L(R)}<$ 0.74 , the spin-current terms of the first and third components dominate in the step because the relative amplitudes of the resonant peaks of the second and fourth channels are negligibly small. Note that with decreasing the spin polarizations of the conduction bands $\left(\delta_{L}, \delta_{R} \rightarrow 1\right)$ the difference in the conduction channels decreases together with TMR.

It is obvious that the top of the flat-dome peaks will decrease showing a steplike behavior with $\delta_{L}$ and $\delta_{R}$ increasing in relation to $\delta_{W}$. The TMR will take on minimal values when $\delta_{L}=\delta_{W}=\delta_{R}$ (note that increasing $\delta_{L}, \delta_{W}, \delta_{R}$ until unity results in decreasing of the spin-polarization value; i.e., magnetic material becomes nonmagnetic).

In conclusion, the shape of the TMR curve depends on the tunneling current, which is a function of the electron trajectories, the thickness of the barriers, and the different 
quantum states of the quantum well. Each conduction spin channel has its own resonant conditions and constraints when electrons move from the majority to the minority subband. These factors are the main reason for the enhanced TMR. Similar regimes of the DMTJ provide a strong spin-filtering effect, which can be controlled by reversing the magnetization of the middle ferromagnetic electrode.

\section{NONRESONANT TUNNEL MAGNETORESISTANCE IN DMTJ}

The results of the previous section are compatible with earlier theoretical data in similar tunnel systems and are complementing them with new effects. This motivated our attempts to apply the developed model to describe experimental data. Most of the experimental data presented in the literature ${ }^{8,11,12}$ correspond to the nonresonant case and do not satisfy the resonant conditions determined in the previous section. The nonresonant tunneling is characterized by the spin-dependent electron transport through the core stack of the DMTJ but without mixing with QW states in the middle layer. In this case DMTJs can be modeled in two ways: (i) DMTJ with coherent tunneling (CHT) and (ii) consecutive tunneling (COT), which is a model that considers the DMTJ as two single-barrier magnetic tunnel junctions (SMTJs) connected in series.

\section{A. DMTJ in coherent tunneling regime}

The normalized TMR $-V_{a}$ curves presented in Fig. 6 are determined by the equation $\mathrm{TMR}=\left(J^{\mathrm{P}}-J^{\mathrm{AP}}\right) / J^{\mathrm{AP}} \times$ $\left(\mathrm{TMR}_{\max }^{-1}\right)$ with $J^{\mathrm{P}(\mathrm{AP})}=J_{\downarrow}^{\mathrm{P}(\mathrm{AP})}+J_{\uparrow}^{\mathrm{P}(\mathrm{AP})}$, where spin-up and spin-down currents can be determined by Eq. (1) and TC is calculated for the entire double-barrier structure by Eq. (6). Equation (1) is applied for positive values of $V_{a}$ while the solution for negative ones can be derived using symmetric relations of the system; i.e., the parameters of the electronic states of the contact electrodes must be reversed: $k_{F \uparrow \text { maj }}^{L(R)} \rightarrow$ $k_{F \uparrow \mathrm{maj}}^{R(L)}, k_{F \downarrow \text { min }}^{L(R)} \rightarrow k_{F \downarrow \text { min }}^{R(L)}$.

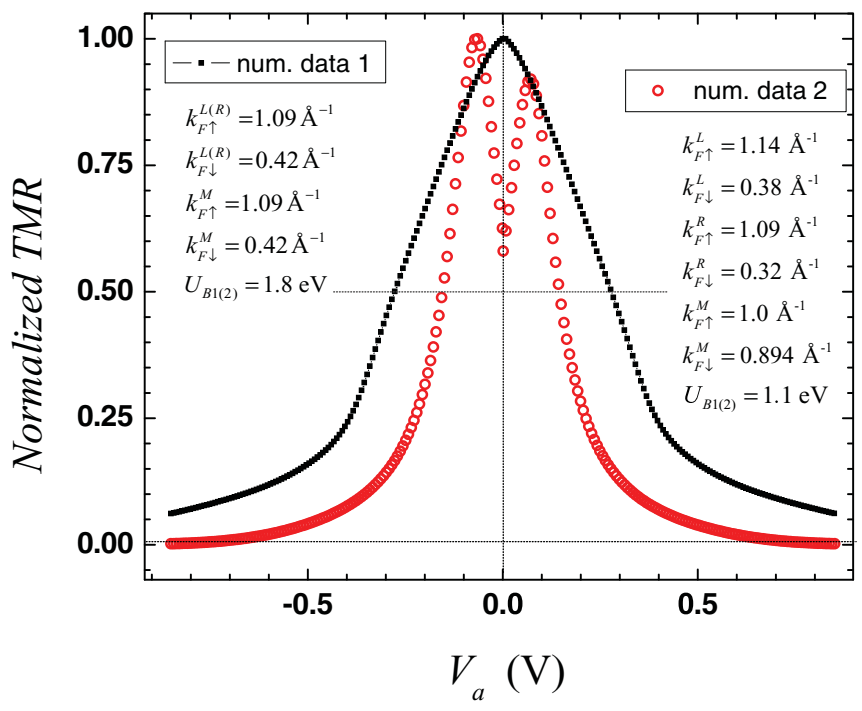

FIG. 6. (Color online) Bias voltage dependence of the normalized TMR of DMTJs in the case of coherent tunneling. $L_{1(2)}=25 \AA$, $L_{W}=10 \AA, m_{L, W, R}=0.8 m_{e}, m_{1,2}=0.4 m_{e}$.

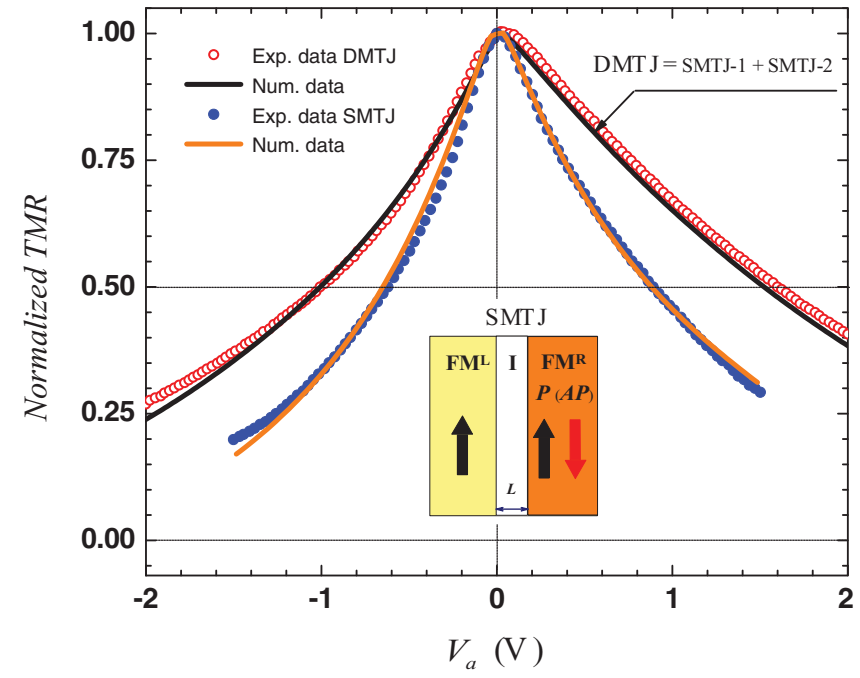

FIG. 7. (Color online) TMR versus voltage for single and double barrier magnetic tunnel junctions in the case of consecutive tunneling (SMTJs connected in series). Numerical parameters for SMTJ: $U_{B}=$ $2.8 \mathrm{eV}, L=23 \AA$; for DMTJ: SMTJ-1(2): $U_{B 1(2)}=2.7 \mathrm{eV}, L=25 \AA$, $m_{1(2)}=0.46 m_{e}, m_{L(R)}=0.76 m_{e}$.

Figure 6 shows examples of two possible behaviors of the DMTJ with a very thin middle layer of $L_{W}=10 \AA$ where coherent tunneling is realized (see numerical data 1 and 2 ). The effect of voltage asymmetry arises in the case of a different initial set of $k_{F, s}^{l}$ for each layer while keeping the condition of equal barriers (see curve 2). As shown, the TMR effect can reach large values for certain values of the voltage. In this small range of voltages, one of the spin channels (which is the first one) has only one peaklike tunnel probability while the other spin channels have no peaks, resulting in the untypical behavior of $J^{\mathrm{P}}$ in relation to $J^{\mathrm{AP}}$. The symmetric case depicted by curve 1 occurs when the wave vectors of the left and right layers are equal for the majority and minority subbands $\left(k_{F \uparrow}^{L}=k_{F \uparrow}^{R}\right.$ and $\left.k_{F \downarrow}^{L}=k_{F \downarrow}^{R}\right)$.

It is worth mentioning that in other cases, obtained by, e.g., varying $L_{1(2)}, L_{W}, V_{01(2)}$, effective masses, or $k_{F, s}^{l}$, other behaviors for CHT can be observed. One of the TMR- $V_{a}$ curves with strong asymmetric voltage branches for DMTJs was derived by Nozaki et $_{\text {al. }}{ }^{12}$ for $\mathrm{Fe} / \mathrm{MgO}(20 \AA) / \mathrm{Fe}(15 \AA) / \mathrm{MgO}(20 \AA) / \mathrm{Fe}$ junctions. We suppose that their results can be classified as CHT because the shape of the DMTJ curve has a similar pattern to our numerical data 1 (see curve 1 in Fig. 6, and DMTJ curve in Ref. 12). This case is difficult to reproduce numerically and experimental data may correspond to mixed regimes of CHT and COT at the same time and may contain an asymmetry of the barrier's heights and thicknesses. However, the shape of the curves is different from those, which can be found in the literature for typical TMR- $V_{a}$ behavior for DMTJ, e.g., Refs. 8,11. Therefore, the CHT representation is not a suitable model to reproduce the most typical TMR- $V_{a}$ behaviors including the asymmetry for positive and negative voltage.

\section{B. DMTJ in consecutive tunneling regime}

Important experimental results were published recently in several papers ${ }^{8,28}$ in which asymmetric voltage behavior (AVB) and the annealing effects of TMR dependencies in 
SMTJ and DMTJ were investigated. Feng et al. ${ }^{8}$ showed experimentally that TMR asymmetry in DMTJ can be model by a serial connection of SMTJs (consecutive tunneling). At the same time the symmetric or nonsymmetric voltage behavior can be used as criteria showing the quality of the deposited layers. ${ }^{11}$

Figure 7 shows normalized TMR- $V_{a}$ curves for the SMTJ and DMTJ where the DMTJ is modeled by junctions connected in series. The results are in good agreement with experimental ones obtained by Feng et al. Asymmetric voltage behavior occurs in spite of the same type of the magnetic materials for $\mathrm{FM}^{\mathrm{L}}, \mathrm{FM}^{\mathrm{W}}\left(L_{W}=30 \AA\right)$, and $\mathrm{FM}^{\mathrm{R}}$ and equal barrier thicknesses $L_{1(2)}=25 \AA$. The criteria applied here for finding the model parameters are the best fit of the normalized TMR to the normalized experimental data as well as $V_{a}=V_{\text {half }}$. The voltages $V_{\text {half }}^{\text {SMTJ }}$ and $V_{\text {half }}^{\text {DMTJ }}$, where the TMR ratio obtains half of its maximum value, are estimated to be $-0.63 \mathrm{~V}$ and +0.89 $\mathrm{V}$ for SMTJ and $-1.0 \mathrm{~V}$ and $+1.5 \mathrm{~V}$ for DMTJ, which are close to experimental ones. The numerical values of the wave vectors were estimated as follows: For SMTJ: $k_{F \uparrow}^{L}=1.29 \AA^{-1}, k_{F \downarrow}^{L}=$ $0.39 \AA^{-1}, k_{F \uparrow}^{R}=1.28 \AA^{-1}, k_{F \downarrow}^{R}=0.44 \AA^{-1}$. For DMTJ: for SMTJ-1, which is the first of the two SMTJs connected in series and for the positive voltage (and for $J_{1}^{\mathrm{P}(\mathrm{AP})}$ calculation): $k_{F \uparrow}^{L}=$ $1.3 \AA^{-1}, k_{F \downarrow}^{L}=0.5 \AA^{-1}, k_{F \uparrow}^{R}=1.028 \AA^{-1}, k_{F \downarrow}^{R}=0.36 \AA^{-1}$; and for SMTJ-2, the second SMTJ (for $J_{2}^{\mathrm{P}(\mathrm{AP})}$ calculation): $k_{F \uparrow}^{L}=1.028 \AA^{-1}, \quad k_{F \downarrow}^{L}=0.36 \AA^{-1}, \quad k_{F \uparrow}^{R}=1.09 \AA^{-1}$, $k_{F \downarrow}^{R}=0.27 \AA^{-1}$. The initial value of the wave vectors for the left side of SMTJ-2 is equal to the one on the right side of SMTJ-1 due to the common middle FM layer. The definition of the normalized TMR is calculated as in the previous case of CHT except of the determination of the current components, which can be written as follows for SMTJs connected in series:

$$
J^{\mathrm{P}(\mathrm{AP})}=\frac{J_{1}^{\mathrm{P}(\mathrm{AP})} J_{2}^{\mathrm{P}(\mathrm{AP})} V_{a}}{V_{a 2} J_{1}^{\mathrm{P}(\mathrm{AP})}+V_{a 1} J_{2}^{\mathrm{P}(\mathrm{AP})}},
$$

$J_{n}^{\mathrm{P}(\mathrm{AP})}=J_{n, \uparrow}^{\mathrm{P}(\mathrm{AP})}+J_{n, \downarrow}^{\mathrm{P}(\mathrm{AP})}$, where $n=1,2$ is the number of the SMTJ in the series connection, and $V_{a 1}, V_{a 2}$ are the voltage drops for the first and second SMTJ, respectively. Considering equal barriers we have $V_{a 1}=V_{a 2}=\frac{V_{a}}{2} \equiv V$ and thus

$$
J^{\mathrm{P}(\mathrm{AP})}=\frac{2 J_{1}^{\mathrm{P}(\mathrm{AP})} J_{2}^{\mathrm{P}(\mathrm{AP})}}{J_{1}^{\mathrm{P}(\mathrm{AP})}+J_{2}^{\mathrm{P}(\mathrm{AP})}} .
$$

The current densities $J_{1}^{\mathrm{P}(\mathrm{AP})}$ and $J_{2}^{\mathrm{P}(\mathrm{AP})}$ are found using Eq. (1) but with the TC derived for a single-barrier structure $D_{s}^{\mathrm{SB}}$. For stand-alone SMTJs it needs to be considered with $D_{s}^{\mathrm{SB}}$ and $V_{a}=V$. In particular, if one of the barrier heights or $L_{W}$ vanished in Eq. (6), it would represent the case of a single-barrier structure (the same results would be achieved if the TC were taken from Ref. 25, where $D_{s}^{\mathrm{SB}}$ was found separately).

Comparing CHT with COT (Fig. 6 and Fig. 7), it should be noted that for the CHT $L_{W}$ is a parameter of strong influence while it is not in the case of COT. However, it can be expected that there is a mixed case of these two types of tunneling which will be more complicated (for example, for the $\mathrm{P}$ case tunneling could correspond to the coherent tunneling while for the AP case could correspond to consecutive tunneling).

The approach of the presented model is obviously capable of accurately describing the AVB in the case of SMTJs and DMTJs and of classifying the type of tunneling. Unfortunately, the model disregards possible leakages such as spin-flip electron processes, charging effects, roughness, and disorder of the junction surfaces and voltage drops at the electrodes. It was found, for example, that factors such as spin-flip processes or disorder ${ }^{17,29}$ can diminish the difference between the $J^{\mathrm{P}}$ and $J^{\mathrm{AP}}$ suppressing TMR values.

We found that a precise fit of the numerical curves for COT is only possible for either the positive or the negative TMR- $V_{a}$ branch and not for both at the same time when keeping the condition of the common middle layer. The fitted data shown in Fig. 7 are compromise values for the initial parameters for which both TMR branches show minimum deviations to the experiment. Thus, the AVB may help to estimate the exact values of Fermi wave vectors as well as the heights of the barriers in real systems.

\section{SUMMARY AND CONCLUSIONS}

Based on the two-band model, we have presented a quasiclassical approach together with quantum conditions for the tunnel current in various tunnel systems such as SMTJ and DMTJ. The TMR was studied in different regimes of tunneling including resonance and nonresonance behaviors. Our calculated data are largely in agreement with theoretical and experimental results for DMTJs and SMTJs.

We found two methods to describe the nonresonant tunneling mechanisms for DMTJs: One is a DMTJ modeled as single coherent tunneling system and the other one is a model that describes a DMTJ as a consecutive tunneling system which consists of two SMTJs connected in series. Both models (CHT and COT) can reproduce asymmetrical voltage behavior of the TMR. Moreover, asymmetric voltage behavior may help to estimate the exact values of the Fermi wave vectors and the heights of the barriers, as well as explain $V_{\text {half }}$ values in real systems. The values of the wave vectors applied for the model are comparable with those that can be found in many experimental or theoretical works. We also showed the importance of zero bias shift $V_{0}$ as well as the momentum conservation law, which apply strict conditions to the electron transport.

One of the advantages of the DMTJ in the resonant tunneling regime is the high value of the resonant tunneling current, which is achieved with rather large widths of both potential barriers, due to QW states of the spin current components. For appropriate voltages the increased TMR values of the stable plateau of the flat-dome pattern can be used for applications. In the case of the single-barrier structure with the same width, the tunnel current and TMR values vanish rapidly.

\section{ACKNOWLEDGMENTS}

This work was supported by King Abdullah University of Science and Technology together with bilateral program RFBR (Project No. 10-02-91225-CT $a$ )-TUBITAK (Project No. 209T061). 
*arthur.useinov@kaust.edu.sa

${ }^{1}$ N. Mott, Proc. R. Soc. London 153, 699 (1936).

${ }^{2}$ I. Campbell, A. Fert, and A. Pomeroy, Philos. Mag. 15, 977 (1967).

${ }^{3}$ J. Mathon and A. Umerski, Phys. Rev. B 74, 140404(R) (2006).

${ }^{4}$ S. Ikegawa, Y. Asao, Y. Saito et al., Jpn. J. Appl. Phys. 42, L745 (2003).

${ }^{5}$ A. Iovan, S. Andersson, Y. Naidyuk, A. Vedyaev, B. Dieny, and V. Korenivski, Nano Lett. 8, 805 (2008).

${ }^{6}$ T. Uemura, S. Honma, T. Marukame, and M. Yamamoto, Jpn. J. Appl. Phys. 43, 2114 (2004).

${ }^{7}$ T. Uemura, S. Honma, T. Marukame, and M. Yamamoto, Jpn. J. Appl. Phys. 43, L44 (2004).

${ }^{8}$ G. Feng, S. van Dijken, J. F. Feng, J. M. D. Coey, T. Leo, and D. J. Smith, J. Appl. Phys. 105, 033916 (2009).

${ }^{9}$ H. D. Gan, S. Ikeda, W. Shiga, J. Hayakawa, K. Miura, H. Yamamoto, H. Hasegawa, F. Matsukura, T. Ohkubo, K. Hono et al., Appl. Phys. Lett. 96, 192507 (2010).

${ }^{10}$ T. Nozaki, N. Tezuka, and K. Inomata, Phys. Rev. Lett. 96, 027208 (2006).

${ }^{11}$ S. Colis, G. Gieres, L. Bar, and J. Wecker, Appl. Phys. Lett. 83, 948 (2003).

${ }^{12}$ T. Nozaki, A. Hirohata, N. Tezuka, S. Sugimoto, and K. Inomata, Appl. Phys. Lett. 86, 082501 (2005).

${ }^{13}$ S. Krishnamurthy, A. Chen, and A. Sher, J. Appl. Phys. 84, 5037 (1998).

${ }^{14}$ X. Zhang, B. Li, G. Sun, and F. Pu, Phys. Rev. B 56, 5484 (1997).

${ }^{15}$ T. Kishi and K. Inomata, J. Magn. Soc. Jpn. 23, 1273 (1999).
${ }^{16}$ M. Wilczynski and J. Barnas, J. Magn. Magn. Mater. 221, 373 (2000).

${ }^{17}$ W. Rudzinski and J. Barnas, Phys. Rev. B 64, 085318 (2001).

${ }^{18}$ M. Chshiev, D. Stoeffler, A. Vedyayev, and K. Ounadjela, Europhys. Lett. 58, 257 (2002).

${ }^{19}$ P. Li and H. Dery, Appl. Phys. Lett. 94, 192108 (2009).

${ }^{20}$ D. Herranz, F. G. Aliev, C. Tiusan, M. Hehn, V. K. Dugaev, and J. Barnas, Phys. Rev. Lett. 105, 047207 (2010).

${ }^{21}$ R. Tsu and L. Esaki, Appl. Phys. Lett. 22, 562 (1973).

${ }^{22}$ A. Useinov, R. Deminov, L. Tagirov, and G. Pan, J. Phys. Condens. Matter 19, 196215 (2007).

${ }^{23}$ D. Serrate, P. Ferriani, Y. Yoshida, S.-W. Hla, M. Menze, K. Bergmann, S. Heinze, A. Kubetzka, and R. Wiesendanger, Nature Nanotechnol. 5, 350 (2010).

${ }^{24}$ H. Oka, P. Ignatiev, S. Wedekind, G. Rodary, L. Niebergall, V. Stepanyuk, D. Sander, and J. Kirschner, Science 327, 843 (2010).

${ }^{25}$ A. Useinov, R. Deminov, N. Useinov, and L. Tagirov, Phys. Status Solidi B 247, 1797 (2010).

${ }^{26} \mathrm{E}$. Kane and E. Blount, in Tunneling Phenomena in Solids, edited by E. Burstein and S. Lundqvist (Plenum, New York, 1969), pp. 9-11.

${ }^{27}$ J. Faure-Vincent, C. Tiusan, C. Bellouard, E. Popova, M. Hehn, F. Montaigne, and A. Schuhl, Phys. Rev. Lett. 89, 107206 (2002).

${ }^{28}$ J. Shin, B. Min, J. P. Hong, and K.-H. Shin, Appl. Phys. Lett. 95, 222501 (2009).

${ }^{29}$ P. X. Xu, V. M. Karpan, K. Xia, M. Zwierzycki, I. Marushchenko, and P. J. Kelly, Phys. Rev. B 73, 180402(R) (2006). 